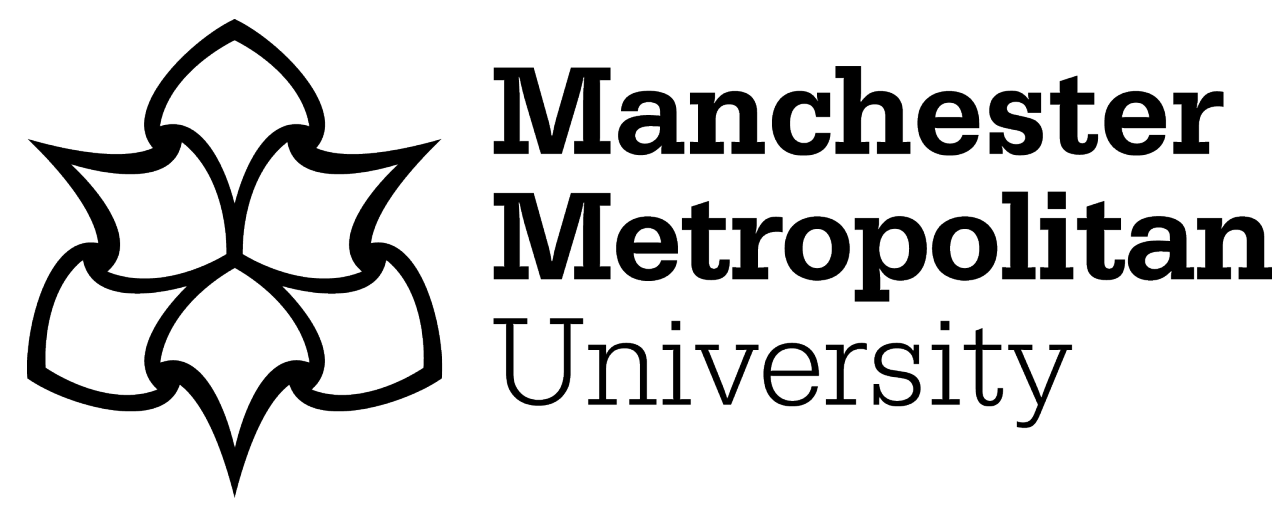

Zemouri, S, Djahel, S and Murphy, J (2019) An Altruistic Prediction-Based Congestion Control for Strict Beaconing Requirements in Urban VANETs. IEEE Transactions on Systems Man and Cybernetics: Systems, 49 (12). pp. 2582-2597. ISSN 2168-2216

Downloaded from: https://e-space.mmu.ac.uk/619213/

Version: Accepted Version

Publisher: Institute of Electrical and Electronics Engineers

DOI: https://doi.org/10.1109/TSMC.2017.2759341

Please cite the published version 


\title{
An Altruistic Prediction-Based Congestion Control for Strict Beaconing Requirements in Urban VANETs
}

\author{
Sofiane Zemouri, Member, IEEE, Soufiene Djahel, Senior Member, IEEE, \\ and John Murphy, Senior Member, IEEE
}

\begin{abstract}
Periodic Beacon Messages are one of the building blocks that enable the operation of VANET applications. In vehicular networks environments, congestion and awareness control mechanisms are key for a reliable and efficient functioning of vehicular applications. In order to control the channel load, a reliable mechanism allowing real time measurements of parameters like the local density of vehicles is a must. These measurements can then serve as an input to perform a fast adaptation of the transmit parameters. In this context, considerable efforts have been directed in the recent years towards designing flexible yet robust protocols solving this problem; yet, very few have considered a proactive adaptation of the transmit parameters as a preventive measure from channel load peaks. To this end, we take the opportunity to introduce $P \& A-A$, a new congestion control protocol that performs a joint adaptation of the transmit rate and power, relying on an altruistic short-term prediction algorithm that estimates the vehicular density around a given vehicle within the next short while. Additionally, P\&A-A adapts the transmit parameters in a way that guarantees the strict beaconing requirements and satisfies the level of awareness required for the operation of most critical VANET applications. The results of the simulations performed in a realistic scenario justify our theoretical considerations and confirm the efficiency and the effectiveness of our protocol by showing significant improvements in terms of network performance (up to $8 \%$ and $14 \%$ improvement in collision rate; and up to $10 \%$ and $20 \%$ increase in busy ratio compared to our previous scheme and the ETSI schemes respectively) as well as the achieved level of awareness (higher coverage with higher transmission rate and power in dense scenarios, and up to $8 \%$ and $55 \%$ improvement in density perception accuracy compared to our previous scheme and the ETSI schemes respectively).
\end{abstract}

Index Terms-Adaptive Beaconing, Transmission Rate Control, Transmission Power Control, Density prediction, Density forecasting, VANETs, ITS.

\section{INTRODUCTION}

$\mathbf{I}$ $\mathrm{N}$ the near future, Vehicular Ad Hoc Networks will be established by enabling wireless communication between vehicles (Vehicle-to-Vehicle) and to the infrastructure (Vehicleto-Infrastructure). Using cooperative traffic applications, vehicular networks promise to solve many of today's road traffic problems such as improving the safety of road users (like intersection collision warning applications, approaching

S. Zemouri and J. Murphy are with the Department of Computer Science and Informatics, University College Dublin, Dublin, Ireland e-mail: sofiane.zemouri@ucd.ie; j.murphy@ucd.ie.

S. Djahel is with the Department of Computing, Mathematics \& Digital Technology, Manchester Metropolitan University, Manchester, UK e-mail: s.djahel@mmu.ac.uk. emergency vehicle, post crash warning...), shortening their trip times (trip planning based on real time feedback from the road infrastructure to avoid congested areas) and enhancing their driving experience (infotainment services like local targeted advertisement or internet access for the passengers). In such networks, characterised by their ad hoc nature, these traffic applications are only made possible thanks to some short singlehop messages compensating the absence of a central entity that monitors the state of the network and keeps track of its structure. These messages, often referred to as beacons (also called BSM Basic Safety Messages in the US or CAM Cooperative Awareness Messages in Europe), are periodically broadcasted by each vehicle in order to provide other participants of the network with information about their surroundings (density, positions, speeds, headings, etc). It is widely accepted in the vehicular networking community that these short messages are crucial for the operation of any kind of application whether a safety or a non-safety one. However, the high dynamicity of this network characterised in the constant movement of vehicles continuously altering its structure, leads to a fast expiration of beacons content. Therefore, the more up-to-date the beacon is, the more accurate the information contained in it is. According to the National Highway Traffic Safety Administration and the Crash Avoidance Metrics Partnership [1], most safety applications cannot guarantee accurate results with a beaconing frequency lower than $10 \mathrm{~Hz}$, while some of them require a frequency up to $50 \mathrm{~Hz}$ to run smoothly and efficiently [2].

These beacons are transmitted on the so-called Control Channel $(\mathrm{CCH})$, which is the central amongst the six other Service Channels (SCH), as defined in the IEEE 802.11p WAVE Standard [3] in the US; and the fifth channel amongst the six SCHs according to ETSI [4] in Europe. These channels are located around the frequency band of $5.9 \mathrm{GHz}$ in both standards, and have a bandwidth of $10 \mathrm{MHz}$ each. This limited channel width translates in a limited transmit capacity. In simple words, within a fixed period of time, only a limited number of vehicles can successfully transmit their beacons. However, this capacity may be reached quickly in highdensity scenarios like urban areas. Moreover, the possible $\mathrm{CCH} / \mathrm{SCH}$ alternation defined in IEEE 1609.4 [5] adds on to the complexity of this problem since it dictates that only half the time is allocated to the $\mathrm{CCH}$ channel in short 50 Milliseconds intervals called CCHI.

These are the main reasons that often lead to conges- 
tion in the channel. Such congestion might have devastating consequences on network performance. In the absence of a proper packet loss recovery mechanism, VANETs cannot afford losing beacons at the risk of compromising the proper operation of safety and critical applications and might even endanger the safety of road users. This problem is known in VANETs as the congestion control problem.

In the recent years, many efforts have been directed towards designing efficient mechanisms to control the load generated by beacons. These solutions mainly focus on one of the two aspects: regulating the periodicity of beacons by adapting their transmit rate, or adjusting their transmit power. The first approach aims to reduce the number of messages inserted in the network, while the second is intended to limit their extent and benefit from the spatial reusability of the channel.

The above approaches can be classified as reactive congestion and awareness control solutions. In other words, whenever congestion occurs in the network, an adaptation of the relevant transmission parameters follows to counterbalance it. The main issue with these approaches is the fact that the transmission parameters adaptation is only triggered after the congestion occurs, but the loss of information inflicted in the time period needed for the system to react cannot be undone. In a highly mobile, unstable network like VANETs, channel load fluctuates and peaks are frequent especially in urban areas where the density of vehicles is relatively high. Despite this, very few have considered a proactive solution to take preventive measures to congestion. Using this approach, the near future channel state is approximated by vehicles and congestion is dealt with before it even occurs.

This paper introduces P\&A-A (Prediction \& Adaptation Algorithms), a proactive congestion control solution for strict beaconing requirements. This protocol relies on real-time density information available to individual vehicles to perform Tx parameters adaptation; but it also relies on a proper prediction of this density to allow for proactive adaptation. The contributions of this paper can be summarised in these two aspects:

- A fast transmit rate and power adaptation algorithm that guarantees marginal collision rate while satisfying the level of awareness required for the operation of most VANET applications,

- A new short-term local density prediction algorithm that enables the estimation of near future channel load in order to prevent utilisation peaks and control congestion on the channel,

The adaptation algorithm proposed in this paper is a substantial enhancement of our previous work published in [6]. The novelty consists in the iterative way of converging towards the optimal Tx parameters that deliver optimal local density. These latter density values were validated both analytically and with simulations, and were found to lead to a low beacons collision rate. In addition, the short-term local density prediction algorithm is another layer that further enhances the congestion and awareness control.

The remainder of this paper is organized as follows: in section II we will introduce the standards related to this subject as well as the most significant related works, sections
III, IV and $\mathrm{V}$ will provide a detailed description of the solution, including a comprehensive analysis of the mechanics that govern the channel conditions, and our inspirations and motivations for this work. The performance evaluation, the simulation scenario and results are presented in section VI, and finally we conclude in section VII.

\section{RELATED WORKS}

As stated earlier in the previous section, numerous works have been proposed to deal with the network overload generated by the periodic transmissions of beacons. These works fall under one of the two categories: rate adaptation approaches and power adaptation approaches. As an example for the first category, ATB [7] proposes to reduce the beaconing rate based on two key metrics: message utility and channel quality. Another example is DynB [8], which follows a similar approach but introduces the effects of shadowing caused by both buildings and cars on the wireless channel load.

The main shortcoming of transmit rate control based solutions is that they do not guarantee the strict messaging requirements specified in standards like IEEE WAVE or ETSI ITS. Due to the limited channel capacity, only a limited number of messages can be carried within one CCHI. In other words, if the density of vehicles in the vicinity exceeds a certain number, it will be no longer possible to grant all vehicles a regular access to the channel without a considerable increase in collision rate. This number has been proved to be less than 30 vehicles according to the works in [6] and [8].

As an example for the second category (i.e. transmit power adaptation approach), [9] selects the transmit power according to the utility of the beacon to be transmitted. The authors in [10] follow a completely different criterion and propose to randomly select the transmit power of vehicles following a given probability distribution. In both works, the authors have shown the potential improvement of this approach in terms of channel load control and the achieved vehicle awareness level, especially in closer ranges. The main issue with this class of solutions, however, is the poor awareness level in further ranges. In fact, the reaction time of drivers proves to be too high for situations where vehicles are speeding up. For example, in a straight unobstructed road where vehicles are moving at their maximum allowed speeds, distances travelled by drivers before reacting to a road hazard or to any other obstacle on the road can be quite high and therefore, the awareness of these vehicles should be extended to further ranges.

Some researchers have proposed hybrid solutions like [11][13] where the transmit rate and power are adapted jointly. Building on these approaches and others, ETSI ITS G5 has released a technical specification introducing DCC [14] (Decentralised Congestion Control). The idea is to combine transmit rate and power adaptations with other kinds of adaptations like the Carrier Sense Threshold. Many efforts have followed like [15] and [16], which mainly focus on analysing the performance of DCC, but the results show many flaws in this early version of the standard. The authors in [17] and [18] proposed an inter-flow coding-based protocol 
to reduce the number of generated packets by $25 \%$ using a backbone of vehicles. They use a Fuzzy Logic approach to combine the various parameters involved in the selection of the backbone. The results show significant improvement in terms of packet delivery ratio, end-to-end delay with a remarkably low protocol overhead.

The works in [19] and [20] propose to take advantage of the temporal as well as the spatial reusability of the channel in vehicular multi-hop communications. The authors in [21] propose to give equal consideration to the congestion control and the awareness control integrating two state of the art protocols namely LIMERIC [22] and PULSAR [23]. The authors in [24] proposed a beacon rate adaptation based on Fuzzy Logic. The work in [25] introduces a distributed rate and power adaptation protocol that relies on context and environment awareness. A more recent work presented in [26] proposes a joint rate and power control using online synchronous control to strike a balance between the overall performance and fairness. In [27], the authors have proposed a joint rate/power adaptation to cope with the wireless channel load problem, by analysing a wide range of $\mathrm{Tx}$ parameters and came to the interesting conclusion that the transmit rate adaptation should depend on the desired channel load while the transmit power adaptation should depend on the target region. However, it is unclear how this target region is defined; besides, there is no guarantee that the strict beaconing frequency requirement will be respected. In our previous work, we proposed a congestion control protocol dubbed SuRPA [6] that achieves an acceptable level of awareness and a low collision rate.

In a different viewpoint, we can classify all the above protocols as reactive protocols. In other words, vehicles using this class of protocols react to an increase in beacon collision rate by adapting their transmit parameters accordingly. In this work, we introduce a new family of protocols in which, the adaptation of the transmit rate and power is performed proactively in a way to significantly prevent increases in the channel load (which leads to beacon collisions). Such an approach can considerably improve network performance in terms of maximizing channel utilisation and minimizing collision rates. In order to achieve this, a prediction of the state of the channel - which can be approximated given the local density of vehicles in the near future - is performed. Vehicle density prediction has attracted considerable attention in the recent years, mainly as a way to design and improve the performance of routing protocols like the work in [28]. Other works used the density prediction as a mean of regulating the vehicular traffic and avoiding traffic jams like the work in [29]. However, very few works have used this density prediction in the context of congestion control. A simple prediction algorithm, which forecasts an increase or decrease in the density based on the proximity to intersections, was proposed in [30]. The evaluation of this work has shown the potential of using such an approach. The results of a similar work presented in [31] also show the potential of such an approach.

Our protocol, named P\&A-A introduces a short-term local density prediction algorithm in order to take preventive actions prior to increases in beacons collisions. This prediction is done in a thorough manner and its output is used as a basis for the joint transmit rate and power adaptation. Our method guarantees minimum collision rate, and a good level of local awareness by securing the strict beaconing frequency requirements. In addition, this work provides a comprehensive study of some network performance metrics like the channel busy ratio and the collision rate, and their correlation. It also discusses how these metrics can be translated into Tx parameters (Transmission Rate and Power) that guarantee good performance of the network.

\section{OVERVIEW OF THE PROBLEM}

\section{A. Assumptions}

The first aim of this work is to prevent beacons collisions by providing a better control of their transmission parameters. We focus on urban vehicular scenarios, which are more prone to traffic collisions, leading in a chain of events to beacons collisions. The maximum speed in this context is limited to $50 \mathrm{~km} / \mathrm{h}$; the relative speed between two vehicles moving in opposite directions can reach up to $100 \mathrm{~km} / \mathrm{h}$ (approximately $28 \mathrm{~m} / \mathrm{s}$ ).

We also assume that each vehicle is equipped with a navigation system that enables positioning and time synchronisation as well as IEEE 802.11p [32] communication technology, a single radio transceiver and computational capabilities. A single transceiver means $\mathrm{CCH} / \mathrm{SCH}$ channel switching is enabled in all vehicles.

Upon the expiration of one CCHI, each vehicle is expected to have received 256 Bytes beacon messages from its neighbours. Four additional pieces of information coded in 4 Bytes are appended to the beacon and are used for the local density prediction. The content of these four new fields is explained further in this paper. The addition to the beacon is possible without increasing its size, since the standard specified in [33] defines the structure of the beacon with fields reserved for application specific uses.

\section{B. Theoretical channel capacity calculation}

As stated earlier in Section I, the limited capacity of the $\mathrm{CCH}$ channel considerably limits the amount of data that can successfully be carried within one CCHI. In this brief analytical study, we give a good approximation of the theoretical capacity of the channel. Since all beacons are fixed in size, we can translate this capacity from data units to an approximate number of accesses to the channel over one CCHI. This latter number is then mapped to the optimal number of vehicles competing for an access to the channel within once CCHI, without causing a channel congestion. This optimal number of vehicles, which we will refer to in the remainder of this paper as "optimal local density", guarantees an access to all vehicles within a certain radio range (i.e. vehicles competing with each other for access to the channel) with a marginal collision rate.

The minimum beacons frequency of $10 \mathrm{~Hz}$ means that each vehicle needs to broadcast one beacon in each CCHI. If we consider a CCHI of 48 Milliseconds (excluding the guard intervals), and given that the maximum channel usage should 


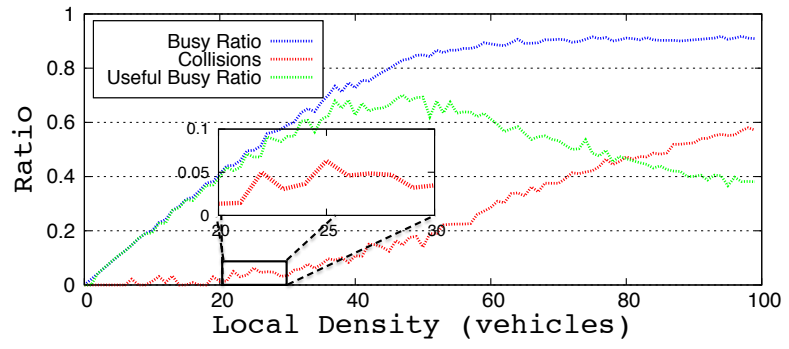

Figure 1: Variation of collision rate, busy ratio and useful busy ratio vs. the local density

not exceed $60 \%$ of the CCHI according to [8], the useful time in one interval can be obtained as follows:

$$
48 \text { Milliseconds } \times 60 \% \approx 28.8 \text { Milliseconds }
$$

If a 256 Bytes beacon transmission takes approximately 1 Millisecond according to [34], the number of vehicles that are allowed to broadcast their beacons within one CCHI is obtained from the following:

28.8 Milliseconds / 1 Millisecond per Vehicle $\approx 28$ Vehicles

This theoretical optimal local density of 28 vehicles is calculated without considering the effect of contention on the channel. In order to consider this effect, we simulated, using NS-3 ${ }^{1}$, a scenario respecting all the above mentioned parameters (10 MHz channels, 48 Milliseconds CCHIs, $10 \mathrm{~Hz}$ beaconing frequency and 256 Bytes beacons). By varying the local density of vehicles from 1 to 100 vehicles, we observed three network metrics, which are the collision rate, the busy ratio and the useful busy ratio. The collision rate was obtained from the following formula:

$$
\text { Collision rate }=\frac{\text { Number of collisions }}{\text { Number of transmissions }}
$$

The busy ratio is obtained as follows:

$$
\text { Busy ratio }=\frac{\sum \text { Transmission times }}{\text { Total CCHI time }}
$$

And finally, the useful busy ratio is obtained using the following formula:

$$
\begin{aligned}
& \text { Useful busy ratio } \\
& =\frac{\left(\sum \text { Transmission times }-\sum \text { Collision times }\right)}{\text { Total CCHI time }}
\end{aligned}
$$

We obtained the graph in Figure 1 depicting where the optimal local density is located with regard to the busy ratio and collision rate levels.

If we consider that the collision rate should not exceed $5 \%$ of total transmissions, we can deduce from the graph an optimal local density in the interval between 22 and 28 vehicles (the area where the busy ratio and the useful busy ratio start going apart), which matches the numbers obtained in the above analytical study.

Starting from this, Tx parameters (transmit rate and power) can be tweaked to adapt the coverage of each individual

\footnotetext{
${ }^{1}$ www.nsnam.org/
}

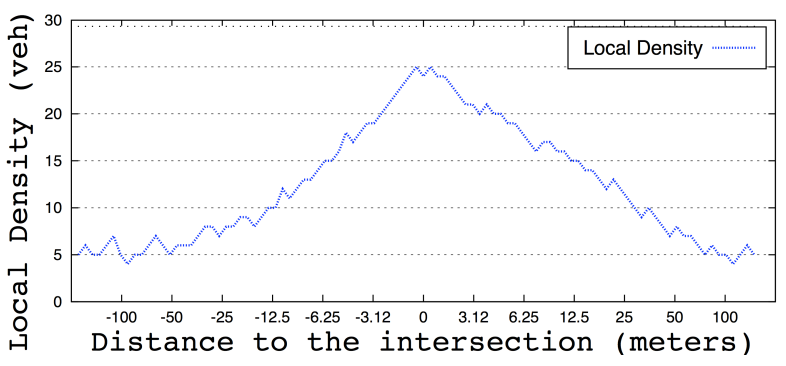

Figure 2: Variation of the local density observed by a vehicle moving towards and crossing through an intersection

vehicle and quickly converge towards the optimal local density (for vehicles within each other's transmission range). Note that this theoretical optimal local density does not depend on external factors like channel conditions or shadowing, achieving the optimal local density does depend on these factors.

\section{Motivations for density prediction}

The main metric that controls whether the Tx parameters should be adjusted upwards or downwards is the local density (observed by each individual vehicle). This metric is read out during fixed 100 Milliseconds intervals called Synchronization Intervals (SI). These readings are then used as a basis for each vehicle individually, to decide how often and how far should its own beacon be broadcasted in the next SI. However, these readings might be outdated by the time they are processed. In fact, a vehicle can only approximate its current local density based on the readings collected in the previous SI. Yet, the high dynamicity that characterises vehicular networks meant that vehicles could observe high variation in their local densities in as little as one SI.

The graph in Figure 2 was obtained by simulating an intersection scenario with traffic lights using NS-3. It shows the high variability in local density observed by a single vehicle crossing through an intersection at a speed of $50 \mathrm{~km} / \mathrm{h}$. Between the moments the vehicle is approximately 6 meters away from the intersection and that when it is in the middle of the intersection, a variation of 13 extra vehicles is observed, which corresponds to more than $50 \%$ increase in its local density. At a speed of $50 \mathrm{~km} / \mathrm{h}$ (approx. $14 \mathrm{~m} / \mathrm{s}$ ), this vehicle travels the last 6 meters before reaching the intersection in approximately 400 Milliseconds. By interpolation, this vehicle observes 3 extra vehicles or $12.5 \%$ increase in its local density every new CCHI, as it gets closer to the intersection.

This is why it is very important that the information used to regulate the flow of beacons (i.e. density of vehicles) reflects the state of the network in the current SI, as opposed to the previously observed state in the last SI. Failing to do this would result in an adaptation of Tx parameters on the basis of biased (outdated) information. According to the specifications introduced in [1], information contained in beacons is outdated after 100 Milliseconds. Under the current assumptions of literature solutions and as shown in Figure 3, this delay can range between 50 and 150 Milliseconds according to the WAVE standard [3] 


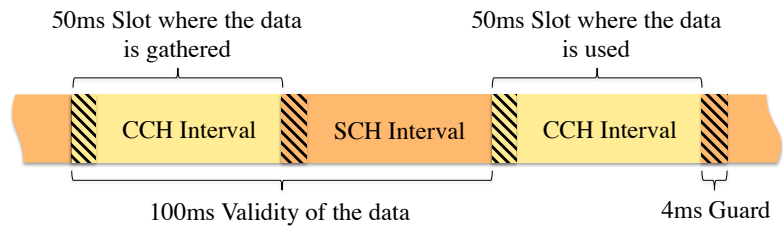

Figure 3: $\mathrm{CCH} / \mathrm{SCH}$ alternation in IEEE802.11p showing the time interval when the data about the density of the network is gathered, and the time interval when it is used (50 to 150 Ms later)

In this context, a short-term prediction of the local density can help improve the transmit parameters adaptation, since it allows it to be performed relying on information that reflects the state of the network at that exact time. As a matter of fact, knowing the current speed and direction of a vehicle, it is pretty straightforward to predict its position within a few Milliseconds or even seconds. Therefore, a short-term prediction of network state (local density of vehicles) is possible in a thorough manner. In addition, this prediction opens up the possibility to take preventive actions by proactively adapting the transmit parameters to the near-future changes in channel conditions. In other words, instead of waiting for the congestion to occur in the channel, the prediction can help avoid it (or at least limiting its severity).

\section{LOCAL DENSITY PREDICTION MECHANISM}

\section{A. Key principle}

For the sake of clarity, the number of the subject vehicle's one hop neighbours will be referred to as "the local density". The area that covers these neighbours is referred to as the subject vehicle's "radio visibility field" or simply "field of view".

An easy approximation of the local density in the near future can be obtained or reported by vehicles that are better positioned to foresee the changes that are going to occur in network topology. As an illustration of this concept, we can think of a vehicle moving towards a congested area. As it gets closer, new vehicles will come into its field of view. This vehicle cannot foresee the increase in density nor can it predict the moment it is going to happen. However, a neighbour vehicle moving ahead can very well report this information as it has a better radio visibility ahead. This very basic principle has been introduced in [30]. However, it can only provide a rough approximation of the short-term local density. In fact, the only information that can be provided by vehicles closer to the congested area using this method is an expected increase or decrease in the local density within the next time interval, but this variation cannot be measured in advance.

This work introduces a more thorough prediction algorithm that enhances the precision of the local density estimation in the near future. The novelty of this work is the use of the local density prediction to improve the network adaptability and performance.

The prediction mechanism is depicted in Figure 4. At time t1 the red car can perceive the grey cars only while the yellow car has a further field of view (up to the blue car). The yellow

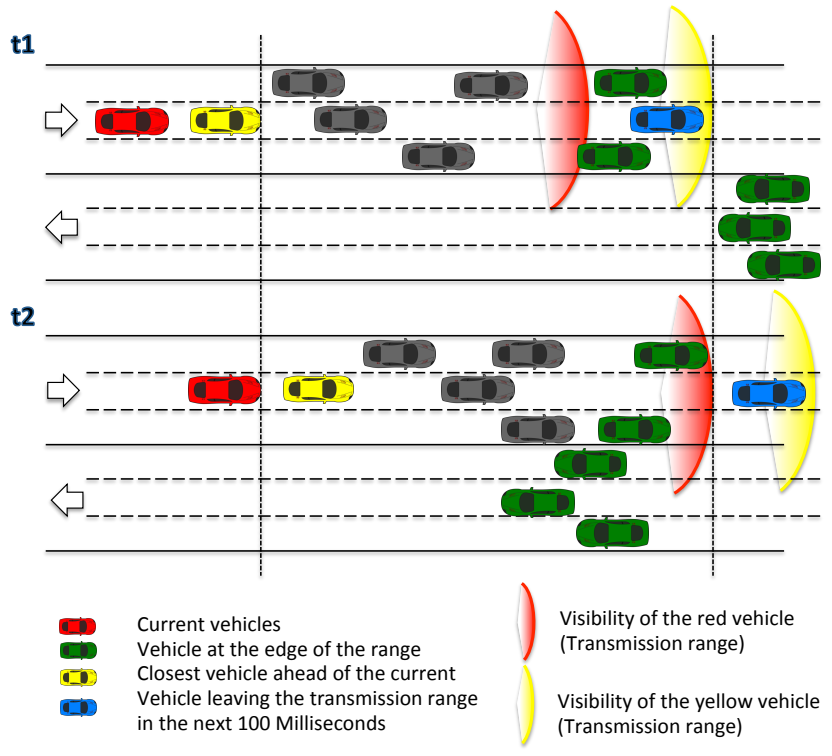

Figure 4: Prediction mechanism illustration with the yellow vehicle making a local density prediction on behalf of the red vehicle

car has then the task of reporting the local density at $\mathrm{t} 2$ to the red car. However at time t1, even the yellow car cannot see the green vehicles that are moving in the opposite direction. In order to make the yellow car aware of these incoming vehicles at $\mathrm{t} 1$, additional information must be reported to it. This information consists in the number of cars that will be overtaken in the opposite direction by the vehicle at the edge of the yellow vehicle's field of view at $\mathrm{t} 2$. Since the blue car is expected to leave this field of view in $\mathrm{t} 2$, the green vehicle right behind it is the best vehicle to relay this last piece of information to the yellow one. This latter vehicle will now have all the necessary pieces to reconstruct the local density ahead for the red car at $t 1$.

The number of vehicles ahead of the subject vehicle (red car) is equal to the current number of vehicles ahead of the closest neighbour ahead (the grey, the two green and the blue vehicles in the same direction) minus the number of vehicles that are going to leave its field of view (blue vehicle); add to it the number of vehicles that are going to overtake the furthest vehicle in the opposite direction (the three green vehicles in the opposite direction). This process is performed to determine the new vehicles ahead as well as the new vehicles behind with regard to the current vehicle's direction. This local density is then included in the yellow vehicle's beacon and broadcasted to be used in time $\mathrm{t} 2$ by the red car.

This mechanism requires the addition of four small pieces of information to beacons, encoded in 4 Bytes. They consist in the following: the first two are the number of vehicles moving in the opposite direction that will be overtaken by the subject vehicle within the next two SIs, as well as the number of vehicles moving in the same direction that will overtake this subject vehicle within the same time interval. The last two are the predicted number of vehicles ahead of the subject vehicle's position and behind it (i.e. as if this current vehicle will not 
move from its current position) within the next SI. Note that each vehicle performs this density prediction on behalf of one of its neighbours, hence the altruistic designation.

\section{B. The proposed prediction algorithm}

In this subsection, we will explain the operation mode of the altruistic short-term density prediction algorithm. Algorithm 1 shows how the prediction is performed, and Table I lists the variables used in it and their designations. This algorithm is node-centric i.e. it is carried out in each individual vehicle and based on the data it has observed and received. Note here that the algorithm is node centric, not to confuse with the prediction mechanism, which is performed collectively among vehicles in a distributed manner.

Table I: Algorithm 1 variable list

\begin{tabular}{cl}
\hline Variable & Designation \\
\hline $\boldsymbol{C} \boldsymbol{V}$ & the Current Vehicle \\
\hline $\boldsymbol{V D A}$ & Vehicle Density Ahead \\
\hline $\boldsymbol{V D B}$ & Vehicle Density Behind \\
\hline $\boldsymbol{C N A}$ & the Closest Neighbour Ahead of the current position \\
\hline $\boldsymbol{C N B}$ & the Closest Neighbour Behind of the current position \\
\hline $\boldsymbol{C N V \boldsymbol { A }}$ & $\begin{array}{l}\text { the Current Number of Vehicles Ahead of current } \\
\text { position }\end{array}$ \\
\hline $\boldsymbol{C N V \boldsymbol { B }}$ & $\begin{array}{l}\text { the Current Number of Vehicles Behind of current } \\
\text { position }\end{array}$ \\
\hline $\boldsymbol{N V A}$ & $\begin{array}{l}\text { the Number of Vehicles Ahead of current position } \\
\text { in the next 100Ms }\end{array}$ \\
\hline $\boldsymbol{N V B}$ & $\begin{array}{l}\text { the Number of Vehicles Behind of current position } \\
\text { in the next 100Ms }\end{array}$ \\
\hline $\boldsymbol{F N A}$ & $\begin{array}{l}\text { the Furthest Neighbour Ahead from the current } \\
\text { position in the next 100Ms }\end{array}$ \\
\hline $\boldsymbol{F N B}$ & $\begin{array}{l}\text { the Furthest Neighbour Behind from the current } \\
\text { position in the next 100Ms }\end{array}$ \\
\hline $\boldsymbol{V O O D}$ & $\begin{array}{l}\text { the number of Vehicles to be Overtaken in the } \\
\text { Opposite Direction in the next 200Ms }\end{array}$ \\
\hline $\boldsymbol{V O S D}$ & $\begin{array}{l}\text { the number of Vehicles that will Overtake the } \\
\text { current vehicle in the Same Direction in the next } \\
\text { 200Ms }\end{array}$ \\
\hline $\boldsymbol{V L T A}$ & $\begin{array}{l}\text { the number of Vehicles that will have Left the } \\
\text { Transmission range Ahead in the next 100Ms } \\
\text { the number of Vehicles that will have Left the } \\
\text { Transmission range Behind in the next 100Ms }\end{array}$ \\
\hline $\boldsymbol{M a x \boldsymbol { D }}$ & $\begin{array}{l}\text { The Maximum Distance a vehicle can travel in one } \\
\text { SI }\end{array}$ \\
\hline
\end{tabular}

At the end of every CCHI, each vehicle computes the number of vehicles that would cross its current offset (distance w.r.t the beginning of the road segment) in both directions (VOOD and VOSD), within the next two SI (200 Milliseconds). These two pieces of information are then included in the beacon. Next, from the beacons previously received from the neighbours, each vehicle will select the beacons received from the furthest neighbour ahead $(F N A)$ and the furthest neighbour behind $(F N B)$ in the next SI with regard to the current position, and retrieve from these beacons the number of vehicles that will have crossed the two respective edges of its field of view in both directions $\left(V O O D_{F N A}\right.$ and $V O S D_{F N B}$ ). Bear in mind that these two pieces of information will be approximately 100 Milliseconds old by the time they are processed, and therefore at this stage, the new

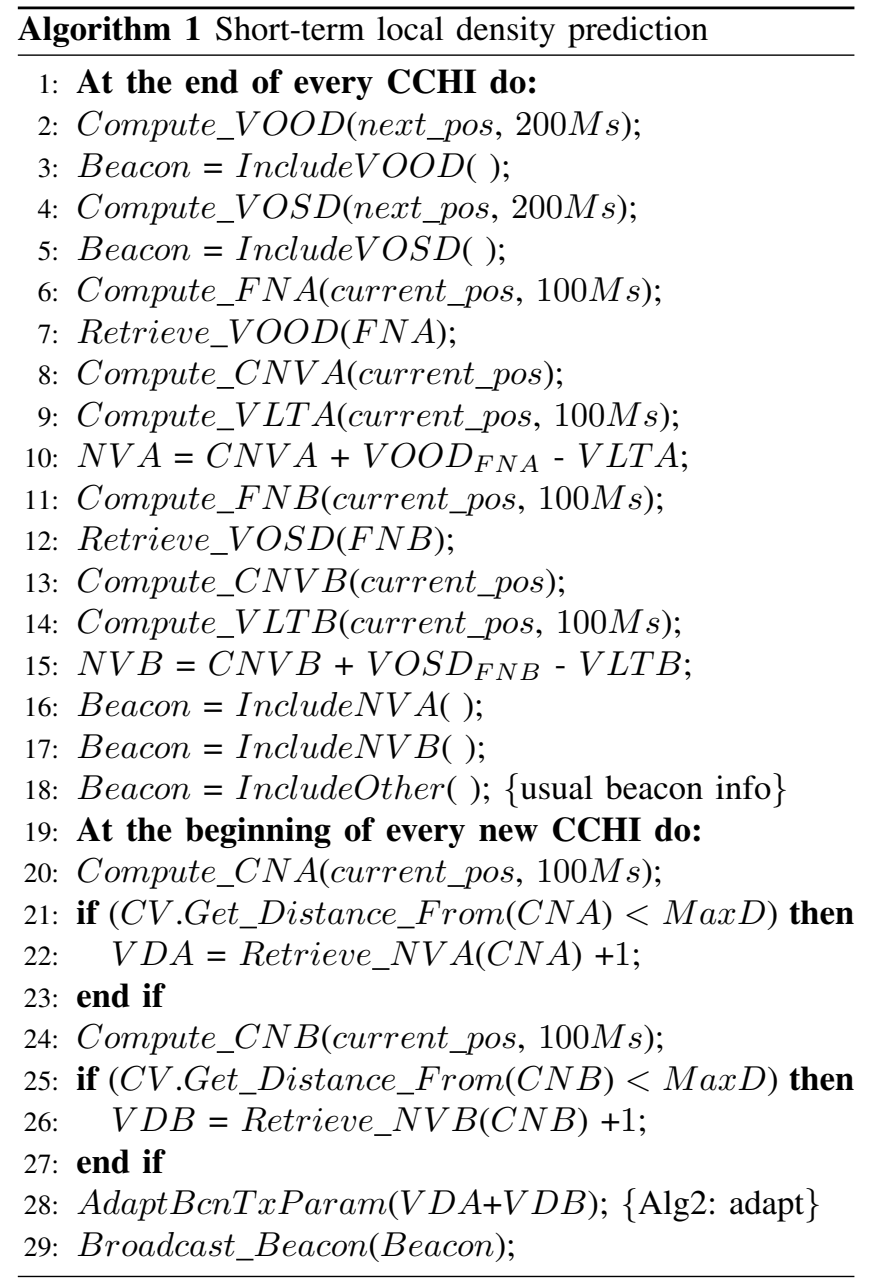

vehicles are expected to cross the edge of the subject vehicle's field of view in another 100 Milliseconds. Each vehicle will now compute the number of vehicles that are expected to leave the field of view ahead and behind (VLTA and VLTB). The predicted local density ahead and behind ( $N V A$ and $N V B)$, for that specific offset within the next SI can then be obtained with the formulas from lines 10 and 15 respectively (see Algorithm 1). These densities are then appended to the beacon and this latter is scheduled to be broadcasted within the next 50 to 100 Milliseconds.

At the beginning of every CCHI and before broadcasting their own beacons, vehicles locate the closest neighbours ahead and behind ( $C N A$ and $C N B$ respectively) and check if they are close enough so that the prediction they provide will be valid. The prediction is valid only if the current vehicle is 100 Milliseconds away from its neighbour. The sum of these two densities (ahead and behind) will constitute the local density prediction for the current vehicle. 1 is always added to the retrieved local density to account for the vehicle performing the prediction.

This prediction scheme is of greater use when enabled close to intersections, where the variation in local densities is greater within a very short time (as shown in 2). However, according to [35], in most major cities, more than $95 \%$ of road segments are shorter than 300 meters with the average road length being 
around 100 meters. Therefore, we chose to enable our density prediction scheme at all times since transmission ranges in VANETs can go even beyond that.

As mentioned earlier, after the local density prediction has been performed and made available to the targeted vehicles, the next step is the adaptation of Tx parameters. The predicted local density serves as the basis for Tx parameters adaptation since, in practice, this latter density is strongly positively correlated with the channel load in that region. The Tx parameters adaptation part is the subject of the next section.

\section{RATE AND POWER ADAPTATION MECHANISM}

\section{A. Key principle}

The problem investigated herein can be summarised in "quickly finding and constantly updating beacons Tx parameters (Transmit Rate TR and Transmit Power TP) that would maximise channel utilisation and minimize the probability of packet collisions". Unlike the authors in [27], We showed that Tx rate adaptation is not the only parameter influencing the channel load, but the power adaptation also has an impact on this load through the spatial dimension of the channel and its reusability. By reducing the target region, fewer vehicles are competing over the channel at every transmission. This not only allows keeping the channel load at reasonable levels in the smaller target region while respecting the strict beaconing frequency requirements in VANETs, but also leaves the channel free for other vehicles to transmit in the further areas. In our previous work [6], we proposed a congestion control solution based on the binary search algorithm. The main idea is to locate "optimal" Tx parameters in the interval of Tx_min and Tx_max using the binary search technique. With a complexity of $\mathrm{O}(\log (\mathrm{n}))$, this algorithm in theory finds an optimal solution in a sufficiently short time. However, with the value of $n$ being too large (Tx power values for example which are in a continuous interval), this solution might not be fast enough and the vehicle might have moved away from the congested area by the time the optimal solution (optimal Tx parameters) could be found.

In this work, we optimised the process of finding a suboptimal solution by considerably narrowing down the search interval [Tx $\left.\min , \mathrm{Tx}^{\cdot} \max \right]$. In simple words, the selection of Tx parameters defines a vehicle's reachable neighbours. The number of these neighbours represents its local density. We have shown in the analytical study presented in Section III-B above, that a local density in the range of $[22,28]$ vehicles will maximise channel utilisation and minimise the collision rate to a marginal value $(5 \%)$.

Our new adaptation algorithm converges much faster to suboptimal Tx parameters values (in one iteration). In case the local density is located outside the above-mentioned range ([22, 28] vehicles), the algorithm adapts Tx parameters in a way to bring this local density to the desired range; then a binary search is performed within the same range to further locate the optimal solution. A single iteration in our algorithm corresponds to one SI, since one new TR/TP value is selected at the beginning of every new CCHI. Note that in a single iteration, our algorithm selects suboptimal Tx parameters
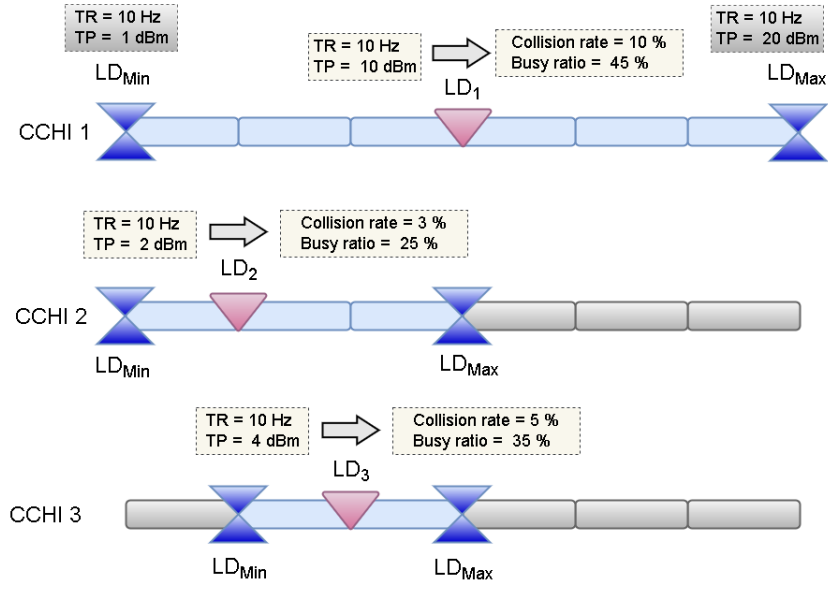

Figure 5: Illustration of the adaptation's key principle: binary search technique applied to VANETs

(that are close enough to the optimal solution), considerably improving channel utilisation and reducing collision rate.

Beyond the faster convergence to the optimal solution and the introduction of the optimal local density concept; the novelty of our protocol lies in its reliance on local density values that reflect the current situation of the vehicular network thanks to the introduction of the new local density prediction algorithm. This way, Tx parameters are proactively adapted to avoid any peaks in channel load and beacons collisions.

Figure 5 demonstrates the binary search concept within the desired range ([22, 28] vehicles) where LD stands for local density. First, LD1 is picked and the corresponding TR/TP are set. At the end of CCHI 1 the collision rate and the busy ratio are measured and processed. In this case, the collision rate is too high, so a lower local density LD2 is picked and the corresponding TR/TP are set. At the end of CCHI 2 the measured collision rate is below the maximum allowed however the channel is under-utilised with a busy ratio of $25 \%$. In this case, a higher local density LD3 is chosen, which satisfies the collision rate constraint while keeping the channel at a good level of utilisation. The methods used in order to select Tx parameters that correspond to a certain local density are explained further in this section.

This algorithm can be optimised using a weighted interpolation. In our case, there is a strong positive correlation between the collision rate and the busy ratio; and between the latter and the picked local density. We leverage this correlation to pick the next local density values. In fact, instead of always picking the middle value in the interval of $L D_{\min }$ and $L D_{\max }$ as the next value; we pick a value that is more likely to narrow down the scope of our search. This value is picked by selecting a weighted factor, which in turn is set based on the Euclidian distance between the current busy/collision ratios and their desired/acceptable values. This weight and the adaptation are also further detailed in the following subsection.

\section{B. The proposed adaptation algorithm}

In order to identify how and when the transmit parameters (rate and power) should be adapted, we asked ourselves these 
three questions: What are each of the above approaches advantages and limitations? what are the most suitable scenarios for their respective usage? and what is the best way to leverage their strengths while overcoming their respective limitations? Without a perfect understanding of each of the approaches limitations', it is very difficult to meet the correct channel load that guarantees a decent packet delivery rate while satisfying the strict awareness level requirement of VANET safety applications.

Our solution combines the rate and power adaptations in a smart way that allows respecting the strict beacons generation frequency requirements of safety applications, while compromising on the transmission power when possible. If the communication overhead increases and, consequently, the collision rate, we first adapt the beacons generation rate in a way to reduce the busy ratio and, therefore, the collision rate. Then, if the $10 \mathrm{~Hz}$ threshold is reached (strict beaconing requirement constraint) and the collision rate is still high, we switch to the transmit power adaptation mode in a way to keep reducing the busy ratio and consequently the collision rate. We refer the reader to our previous paper [6] for the rationale behind this combination.

The main three parameters on which relies the beacons Tx parameters adaptation in our scheme are the local density, the channel busy ratio and the collision rate as all four (including Tx parameters) strongly depend on each other. The local density is obtained by means of a prediction in our scheme (see Section IV); then, the expected busy ratio is inferred from the predicted local density. The rationale for this is simple: considering that each vehicle is expected to broadcast one or more beacons every CCHI, an increase or decrease in the density of vehicles will be accompanied with a proportional variation in terms of the channel busy ratio. In case no prediction is available to a vehicle, the latest available local density is used, which corresponds to the number of beacons received by unique vehicles within the last time interval. In this case, the busy ratio that corresponds to this local density can be retrieved by sensing the channel and adding up the time periods each vehicle spent in the state receive (see equation 4).

The collision rate is retrieved relying solely on the restricted knowledge of the network vehicles have. In fact, collisions are difficult to detect from a vehicle's perspective. One possible solution is the use of the information contained in the sequence control field of the 802.11 MAC header. While it is not possible for a vehicle to detect if its own beacon collided with another beacon from another node, this method allows vehicles to estimate the number of lost beacons sent to them over a period of time. Practically speaking, collisions are read out by checking the beacons sequence numbers on the MAC header, if the sequence $\{3,4,8,9\}$ is received by a vehicle, it will conclude that beacons $\{5,6,7\}$ were lost. This method allows our protocol to have a good estimate of the local channel conditions in order to take the necessary actions. It has previously been used in many works including [36].

Since our adaptation is made based on the three abovementioned parameters (namely the collision rate, the busy ratio and the local density), we set an order of priority

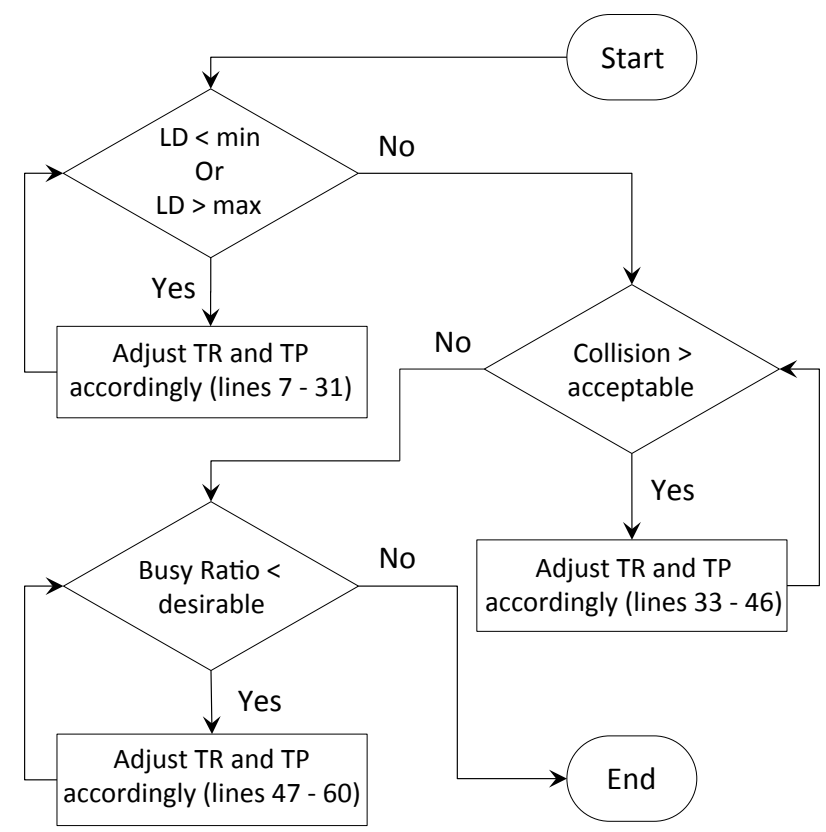

Figure 6: The three main components of Algorithm 2 and their execution sequence

between these three parameters. In other words, which of the acceptable/desirable ratios/values we try to achieve first. The local density has the highest priority for the simple reason that achieving a reasonably low local density prevents from packet collisions (which was demonstrated in subsection III-B) since the two are directly correlated. Collision rate has the second highest priority in our algorithm on account of the devastating effect of packets loss on the performance of any safety or non-safety application in vehicular networks. And finally, maximising the channel utilisation is the least critical and therefore we only attempt to achieve a reasonable busy ratio if the local density is included in the desirable interval and the collision rate is under a certain threshold.

Algorithm 2 shows how our adaptation is performed. This algorithm is executed at the end of each CCHI and is nodecentric i.e. the adaptation is carried out in each vehicle and based on the channel conditions observed at a vehicle level. Figure 6 shows the sequence of execution with respect to each of the parameters (Local Density, Collision Rate, and Busy Ratio). Tx parameters are adapted according to the observation of these three parameters in the order presented.

First, we check if the predicted local density falls into the interval between the minimum and the maximum desired local densities. If it is outside this range, the required adaptation of TR/TP is performed according to the predicted local density. If the local density is already in the range, the collision rate is the next parameter the algorithm checks. If the current collision rate indicates that it is higher than the acceptable collision rate, we should decrease either the transmit rate or the transmit power according to what is available to be adapted. On the other hand, if the collision rate is below the acceptable collision rate, we can look at the busy ratio and in this case increase our selected Tx parameter to increase the 


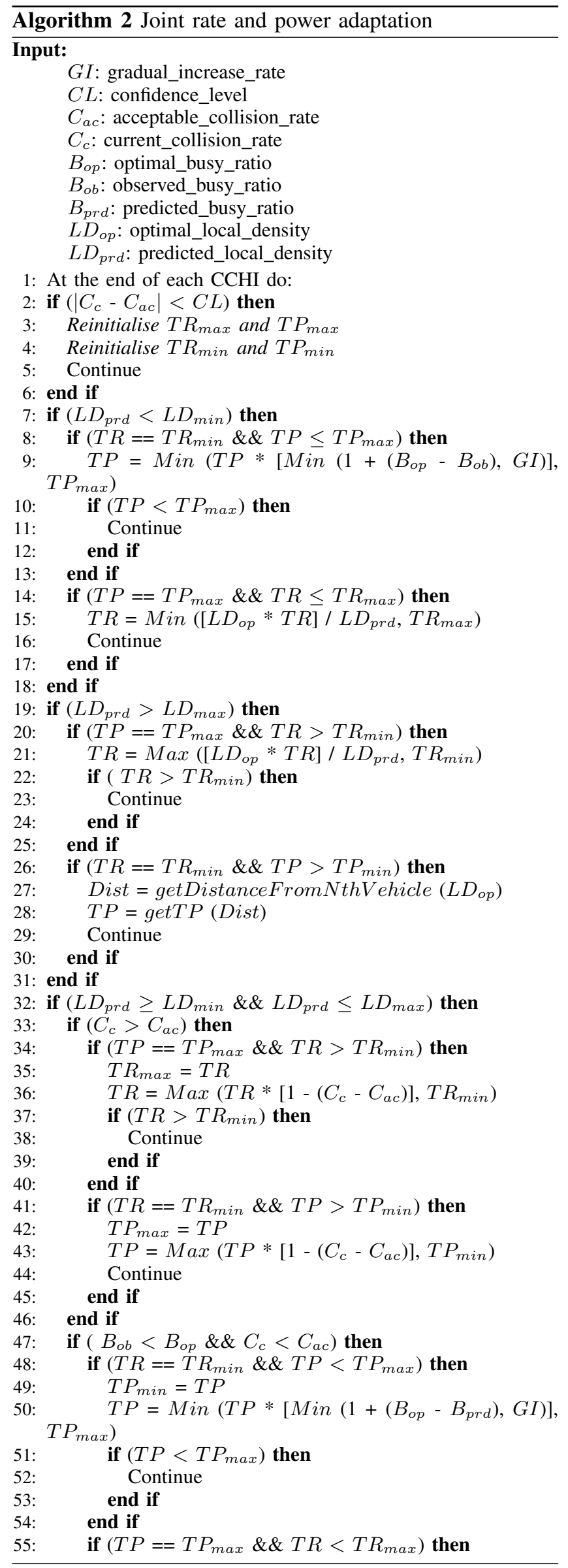

busy ratio if it is lower than the optimal rate. Once the decision about increasing or decreasing Tx parameters has been made, we look at TR and TP values to pick which one of them should be adapted. For example when adapting Tx parameters downwards, if TR can still be adapted, we reduce it first. On the other hand, if TR has already reached its minimum allowed value, TP is adapted.

The third step consists in adapting Tx parameters according to the above conditions. If the predicted local density is in the desired range, the adaptation is made by increasing or decreasing the selected Tx parameter by a certain amount at each step (CCH Interval). This amount is determined using the Euclidian distance between the current channel conditions (i.e. collision rate and channel busy ratio) and the acceptable/desired values. Equations in Algorithm 2 show the adaptation made on TR by decreasing and increasing it in lines 35 and 56 respectively; and TP as well by reducing and expanding it in lines 42 and 49. On the other hand if the predicted local density is outside of the desired range, the adaptation is made by increasing or decreasing Tx parameters based on different methods. In the case of a TR adaptation, this latter is determined using a simple rule of three as specified in lines 14 and 20. For example, if the predicted local density value is 12 vehicles broadcasting at a TR of one beacon every time interval; in order to increase the local density to the equivalent of 24 vehicles we simply have to multiply the current TR by $(24 / 12)$.

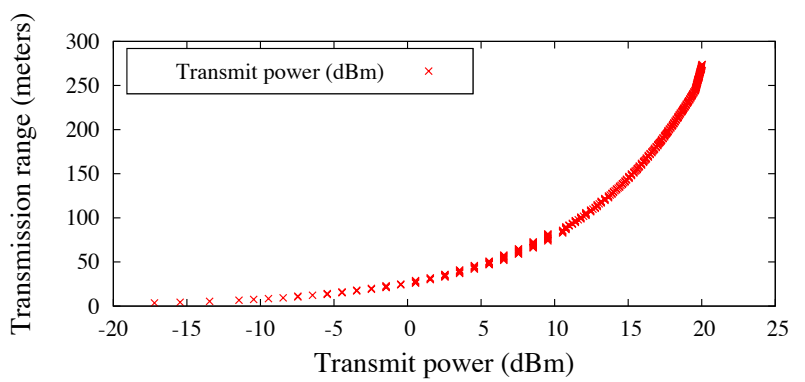

Figure 7: The effect of transmission power on the transmission range in IEEE 802.11p standard

If TP was to be adapted upwards, the Euclidian distance method between the optimal busy ratio and the predicted one is used since there is no direct way to increase the transmission power and reach an exact number of vehicles in the absence of information about the topology of the network beyond the vehicle's own field of view. If this adaptation had to be done downwards, the network topology is known to the vehicle in which case it is possible to reach a subset of vehicles within 
a vehicle's own field of view. In fact, for a given transmission power, vehicles are reached within an easily approximated distance. Figure 7 was obtained by means of simulations using the CORNER ${ }^{2}$ propagation model [37] and shows the distances reached with regard to the TP value in a Line Of Sight (LOS) scenario. Since the goal here is to decrease TP and restrict the geographic area that will be covered by the beacon, only the maximum distance is taken into account. This is why the Non-Line Of Sight scenario is not considered for this matter. The downward adaptation of TP performed in lines 26 - 27 relies on this concept and the data presented in the graph.

The upper and lower bounds $\left(T R_{\max } / T R_{\min }\right.$, $\left.T P_{\max } / T P_{\min }\right)$ correspond to a given temporal local density and are updated at the end of each CCHI right before the adaptation is performed, by setting the previous corresponding Tx parameters as a new bound. These bounds are reinitialised with the maximum/minimum TR/TP values (corresponding to the bounds of the desired local density range) if the difference between the current and the acceptable collision rate is smaller than an amount CL (Confidence Level) that we set beforehand. This means that the nearoptimal Tx parameters have been found and the adaptation is skipped in that step. Note that TR and TP increase is always capped by a gradual increase rate (GI) at each step. This is done to provide a slow increase of the channel busy rate and avoid overloading the channel after an excessive increase of Tx parameters that would compromise the collision rate.

\section{Vi. Performance eVAluation}

In this section, we aim to demonstrate the efficiency of each of the components forming P\&A-A, namely, the altruistic local density prediction mechanism and the joint rate and power adaptation algorithm. To do so, we compared our protocol with four other schemes namely P-SuRPA (a combination of the prediction mechanism introduced in [30] and the adaptation mechanism presented in [6]), and three variants of DCC (Decentralised Congestion Control), which are ETSI-TRC (Transmit Rate Control only), ETSI-TPC (Transmit Power Control only) and ETSI-DCC (combined TRC and TPC). We refer the reader to [14] for more information about DCC. The metrics we picked in order to evaluate the efficiency of our protocol are the collision rate and the busy ratio. A collision is retrieved from the simulation environment if two or more vehicles within each other's range, broadcast their beacons at the exact same timestamp. This information can be easily retrieved from the simulation for each vehicle and the collision rate for any vehicle is calculated as the ratio of the number of collided beacons over the number of all broadcasted beacons within a specified period of time. The overall collision rate is then obtained from aggregating collision rates from all vehicles in the simulation. Similarly, the busy ratio is calculated as the fraction of time the channel is busy from a single vehicle's perspective, within a specified period of time. The overall busy ratio is obtained from the aggregated busy ratios of

\footnotetext{
${ }^{2}$ The choice of this propagation loss model is motivated further in the next section
}

all vehicles in the simulation. The performance evaluation is carried out in three steps: the accuracy of the local density prediction is demonstrated alone by showing how close is this prediction from the real local density compared to the four other schemes; then the benefit from applying the new joint rate and power adaptation is proven by measuring, comparing and interpreting Tx parameters for the same schemes; and finally, the two mechanisms (prediction and adaptation) are tested together against the above mentioned schemes to show the substantial gain obtained after applying P\&A-A. Two scenarios with two different road patterns (road maps imported from OpenStreetMap ${ }^{3}$ ) were selected to carry out the simulations, consisting in a $2 \mathrm{~km}^{2}$ road portion of Manhattan (Figure $8(\mathrm{a})$ ), and a $12 \mathrm{~km}^{2}$ portion of the city of Kirchberg, Luxembourg (Figure 8(b)).

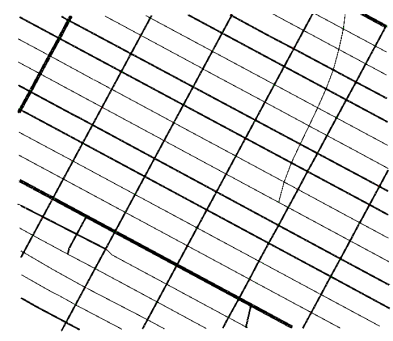

(a) Manhattan map

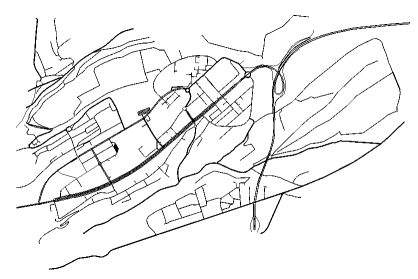

(b) Kirchberg map
Figure 8: A road map portion of (a) Manhattan and (b) the city of Kirchberg, Luxembourg, converted to SUMO format and used in the simulations

One important thing to bear in mind throughout this section is the difference between the "density" and the "local density" as the former represents the density of all vehicles in the simulation at a given time $t$ while the latter stands for the density of vehicles around a given vehicle and as perceived by this vehicle at any given time $\mathrm{t}$.

\section{A. Simulation setting}

We took a special care to conduct our test study in a realistic simulation environment. For a realistic vehicular mobility model, we used HINTS, a platform that couples the microscopic road traffic simulator $\mathrm{SUMO}^{4}$ with the network simulator NS-3, and allows them to exchange information about vehicle movements at runtime. We refer the reader to [38] for detailed description of the HINTS platform operation mode. All vehicles in the simulations move according to the standard SUMO Krauss driver model. We varied the density of vehicles in the simulations from low densities to high densities. The traffic was generated using a SUMO tool called DUAROUTER. This tool allows generating realistic vehicular mobility traces by assigning Origin-Destination pairs based on some statistical data about different areas of the road map (like the population, the age groups, as well as the working population and others), then and computing trips for individual vehicles. The total number of vehicles over the duration of

\footnotetext{
${ }^{3}$ www.openstreetmap.org

${ }^{4}$ www.sumo.dlr.de
} 
the simulation in our experiments is 3200 and 4000 for the Manhattan scenario and the Kirchberg scenario respectively. Note that all densities in this section colorredrepresent the density at a given fraction of time and are expressed in vehicle per kilometre $(\mathrm{veh} / \mathrm{km})$. A kilometre colorredhere is a linear kilometre comprising a two lanes road. We also used a version of the CORNER propagation loss model described in [37], which takes the multi path fading into account, and selectively applies the Rician fading for LOS and Rayleigh fading for NLOS scenarios to capture the shadowing effects caused by buildings. Regarding the shadowing caused by vehicles, it has been shown in [8] that this shadowing is marginal in urban scenarios, and no significant difference is observed between the scenarios where the shadowing caused by buildings only is considered and the scenario where both vehicles and buildings are taken into account. Hence our choice of the CORNER model.

We implemented P\&A-A on each NS-3 node. These nodes have only access to the environmental information the OBU of an actual vehicle has access to (like their own real time position, direction and speed in our case). This information is retrieved by each NS-3 node in real time from SUMO and included in beacon messages. Each vehicle runs P\&A-A algorithm using the restricted knowledge it acquired from the beacons received from its neighbours at each Synchronisation Interval (SI), and only bases its adaptation decisions on this information. Using this simulation model, we made sure to mimic and stay true to the integrity of a real world VANET scenario.

Table II: Simulation parameters

\begin{tabular}{ccc}
\hline & Frequency band & $5.9 \mathrm{GHz}$ \\
PHY & Bandwidth & $10 \mathrm{MHz}$ \\
& Transmission range & $260 \mathrm{~m}$ \\
& Max/Min Tx Power & $20 / 5 \mathrm{dBm}$ \\
& Receiver sensitivity & $-95 \mathrm{dBm}$ \\
& Noise & $-105 \mathrm{dBm}$ \\
\hline \multirow{3}{*}{ LLC } & Bit rate & $6 \mathrm{Mbits} / \mathrm{s}$ \\
& CW & {$[15,1023]$} \\
& AIFSN & 2 \\
\hline \multirow{3}{*}{ ETSI } & Relaxed/Active/restrictive & $20 / 15 /-10 \mathrm{dBm}$ \\
& Relaxed/Active/restrictive & $25 \mathrm{~Hz} / 2 \mathrm{~Hz} / 1 \mathrm{~Hz}$ \\
& Max /Min Channel load & $0.4 / 0.15$ \\
\hline \multirow{5}{*}{ P\&A-A } & Optimal local density & $25 \mathrm{Vehicles}$ \\
& Data message size & $256 \mathrm{Bytes}$ \\
& Max/Min beacons freq & $50 \mathrm{~Hz} / 10 \mathrm{~Hz}$ \\
& Acceptable collision rate & 0.05 \\
& Desired channel load & 0.35 \\
& Gradual increase rate & 1.2 \\
& MaxD & $2 \mathrm{~m}$ \\
\hline
\end{tabular}

For our simulations, we used the IEEE 802.11p Medium Access Control model (MAC) with $10 \mathrm{MHz}$ wide channels according to the WAVE standard [3] and implemented the alternation mechanism between the $\mathrm{CCH}$ and the $\mathrm{SCH}$ channels according to the IEEE 1609.4 specifications [5]. Furthermore, we only used one access category (AC) AC_BE since all beacons use the same AC [4], [33]. We chose a data rate of $6 \mathrm{Mbit} / \mathrm{s}$ [39] and starting values of $20 \mathrm{dBm}$ for the transmit power and $-95 \mathrm{dBm}$ for the receiver sensitivity value to reach approximately 260 meters in LOS [37], and $10 \mathrm{~Hz}$ to $50 \mathrm{~Hz}$ for

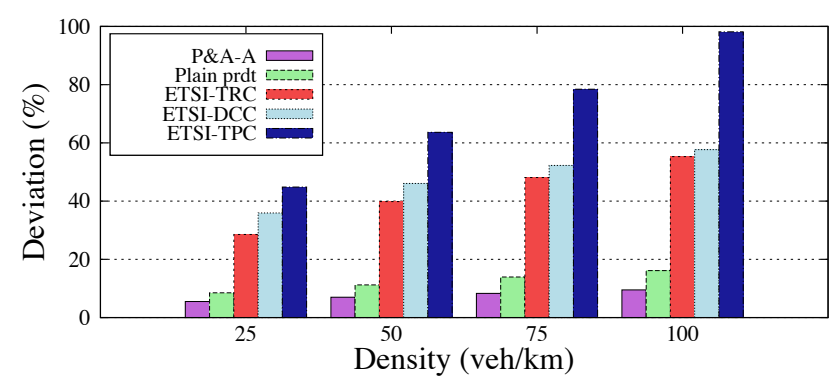

(a) Manhattan

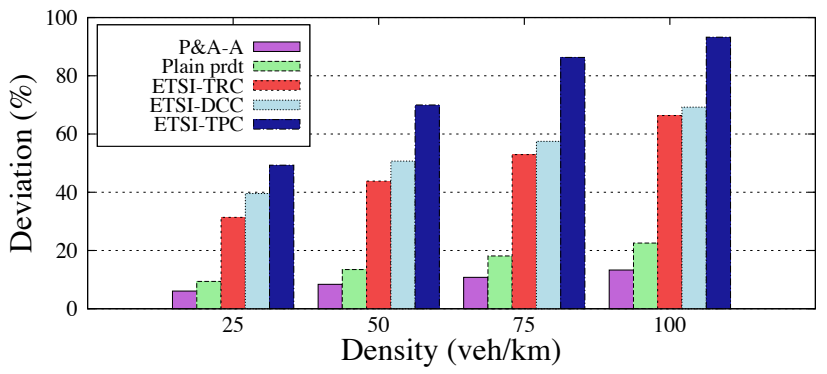

(b) Kirchberg

Figure 9: The percentage of deviation from the effective (real) case under different vehicle densities: P\&A-A vs. plain prediction and ETSI schemes

the beaconing frequency [1], [2]. Note that the transmit rate and transmit power values are the maximum values and are adapted dynamically in our scheme based on the predicted local density and the channel conditions. We set the same starting transmit power values for the ETSI schemes [4] as well to achieve the maximum desired transmission range that is representative of vehicular networks [40] . In addition, DCC schemes were implemented with $\mathrm{CCH} / \mathrm{SCH}$ alternation for fair comparison between these latter schemes and our schemes. P\&A-A specific parameters such as MaxD and the gradual increase rate are tuned after performing some experimental runs and obtaining the optimal values for our scenario. The full list of simulation parameters for P\&A-A as well as for DCC is given in Table II.

Building on this simulation configuration, we implemented our protocol P\&A-A as well as the four above-mentioned schemes and performed several runs of 1800 seconds to obtain more accurate results.

\section{B. Short-term local density prediction evaluation}

In order to showcase the efficiency of our new local density prediction scheme, we compared it with the three ETSI schemes mentioned earlier in this section as well as with the simple prediction scheme presented in [30] that we refer to as plain prediction. The first metric is the deviation of the observed/predicted local density from the real local density (Figure 9). Each vehicle in the simulation observes (and predicts in our scheme) its local density, which is then compared with the effective (real) local density to show the amount of deflection from this real case. A deviation of $0 \%$ is the best case scenario and the higher the deviation the worse 


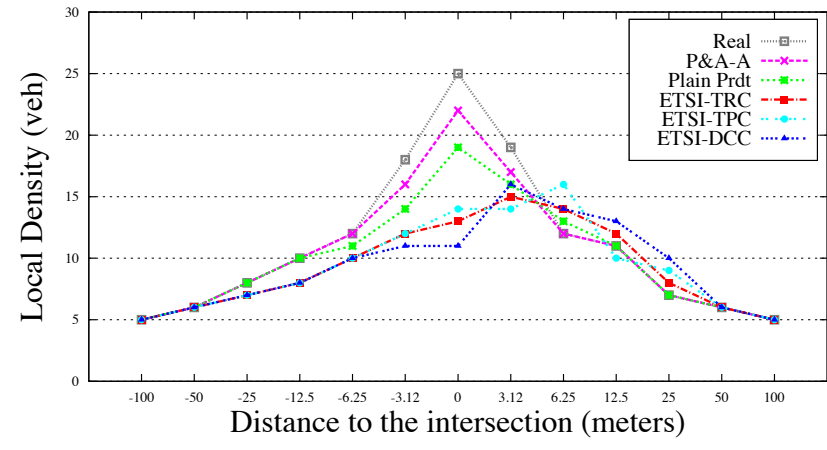

Figure 10: Impact of the distance to the intersection on the observed density: P\&A-A vs. plain prediction and ETSI schemes

the results are. This deviation is calculated using the following formula:

$$
\text { Deviation }=100-100 \times\left(\frac{\sum \text { observed_local_density }}{\sum \text { real_local_density }}\right)
$$

Where the observed local density is the one observed (predicted in our case) by each individual vehicle, and the real local density is the effective local density retrieved from our simulation, which considers a collision free medium. The results shown in figure 9 are obtained using the mean value of all vehicles' local densities in the simulation, and retrieved for different vehicular densities (the total density of vehicles in the simulation as opposed to the local density). The simulations were conducted using the two road maps of Manhattan (Figure 9(a)) and Kirchberg (Figure 9(b)).

This first set of results show a clear advantage for our prediction scheme compared to the other schemes with a deviation at a density of $25 \mathrm{veh} / \mathrm{km}$ of $5 \%$ in the Manhattan scenario and $6 \%$ for Kirchberg map; slightly better than the plain prediction with almost $10 \%$ against $30 \%$ and more for the ETSI schemes (with a peak of to approximately $45 \%$ for ETSI-TPC in both scenarios). The same pattern is observed as the density of vehicles increases in the simulation, the gap gets bigger as we recorded $10 \%$ and $12 \%$ deviation for our scheme at a density of $100 \mathrm{veh} / \mathrm{km}$ for Manhattan and Kirchberg respectively against $17 \%$ and $22 \%$ for the plain prediction and up to $98 \%$ for ETSI-TPC. The degradation in the deviation with the increase of the total density of vehicles in the simulation observed in all the schemes (with different amplitudes) is due to the increase in beacons error rates, as the lost beacons would affect the accuracy of the local density measurement (prediction in our case). Our scheme scales up much better since it also reduces the collision rate, which we will discuss later in this section.

Figure 10 shows the benefit we obtained using our scheme in terms of the observed local density (predicted in our case) from a single vehicle's perspective. We retrieved this observed/predicted local densities from vehicles moving towards an intersection and crossing through it; and compared our results with the four other schemes as well as the real local density that we retrieved from the simulation. Here again, our density prediction scheme performs much better than the four other schemes, as it is the closest to the real density, especially in the area closer to the intersection where the variation of the density is faster and more important. Also, we notice that the plots representing the non-density-based schemes are all slightly skewed to the right compared to the prediction-based schemes. This reflects the up-to-dateness of the data since a slight skewing to the left means in this case that vehicles are perceiving a local density representing an older state.

This metric, like the first one, is meant to show the accuracy of our short-term vehicular density prediction scheme in both a macroscopic point of view in the first (a measure of the overall preciseness of the prediction) as well as a microscopic one in the second (a measure of the preciseness at a vehicle level). The results show a substantial improvement in terms of preciseness and up-to-dateness of the local density with regard to the effective case compared to the other schemes. It is worth to call to mind the importance of an accurate vision of the network. An outdated or biased density observed by vehicles would result in an erroneous adaptation of the transmission parameters, which will in turn cause poor network performance like high packet losses and misuse or under-utilisation of the available bandwidth. This leads us to our next point: the evaluation of the joint rate and power adaptation.

\section{Evaluation of the joint beacon rate and power adaptation}

In this subsection, we show the variation of $\mathrm{Tx}$ parameters (Transmission Rate and Transmission Power) versus the density of vehicles using P\&A-A against SuRPA (without the prediction [6]) and the ETSI schemes. Figure 11 shows the variation of beacons' transmit rate and power for Manhattan and Kirchberg road maps. The graphs in 11(a) and 11(b) show a gradual decrease in the beacons transmit frequency for our scheme as opposed to the ETSI schemes. In addition, our scheme does not go below the limit of $10 \mathrm{~Hz}$, performs slightly better than SuRPA and stabilises at density values around 55 $\mathrm{veh} / \mathrm{km}$ in the first scenario and $65 \mathrm{veh} / \mathrm{km}$ in the second against $45 \mathrm{veh} / \mathrm{km}$ and $55 \mathrm{veh} / \mathrm{km}$ respectively for SuRPA. The two other schemes (ETSI) go down to $1 \mathrm{~Hz}$ as the density of vehicles increases and the communication overhead with it. In Figures 11(c) and 11(d), the transmit power for our scheme is stable and at the maximum value since, up to a density of $55 \mathrm{veh} / \mathrm{km}$ for Manhattan scenario and $65 \mathrm{veh} / \mathrm{km}$ for Kirchberg, only the rate adaptation is activated. Beyond these two respective densities, the transmit power starts to decrease gradually to reach the values of $-3 \mathrm{dBm}$ and $5 \mathrm{dBm}$ in the two scenarios respectively. Our scheme performs better than SuRPA for which, the transmit power drops to $-6 \mathrm{dBm}$ and $0 \mathrm{dBm}$ respectively at a density of over $100 \mathrm{veh} / \mathrm{km}$. The ETSI schemes on the other hand drop sharply and reach the bottom value of $-10 \mathrm{dBm}$.

This set of results reveals two important things: first, the gradual evolution of $\mathrm{Tx}$ parameters in our scheme means a better adaptation and adjustment to the channel conditions. Second, the higher values of our Tx parameters means a more up-to-date context information for vehicles, shown in 11(a) and 11(b) as beacons are exchanged more frequently; and a higher level of awareness shown in 11(c) and 11(d) since 


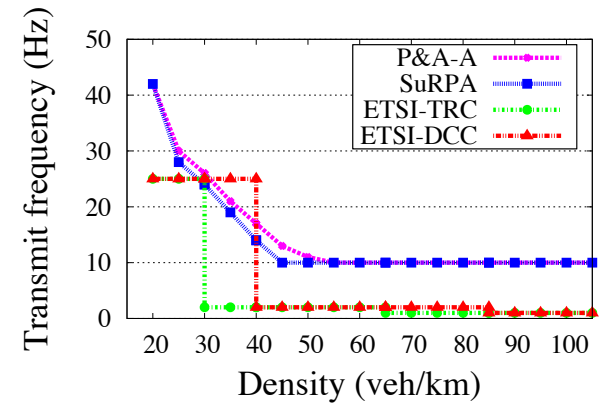

(a) Manhattan

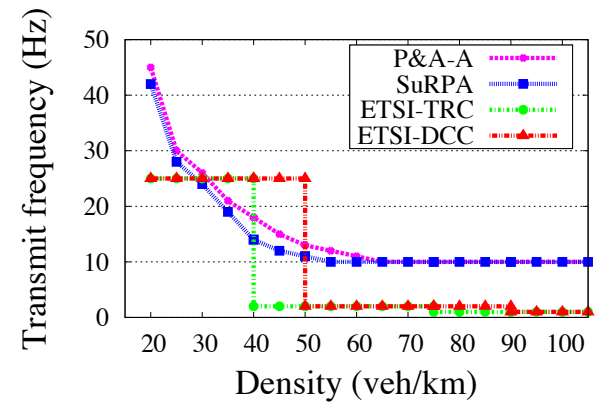

(b) Kirchberg

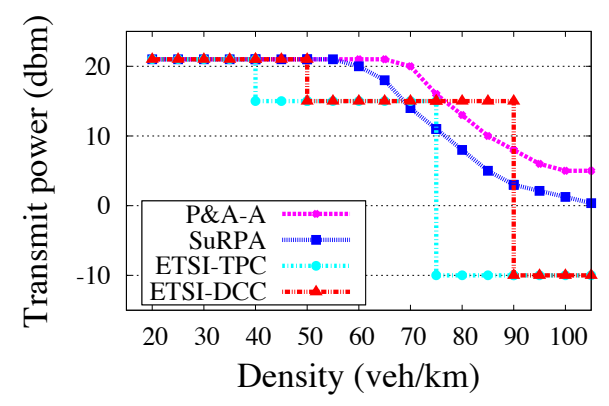

(d) Kirchberg

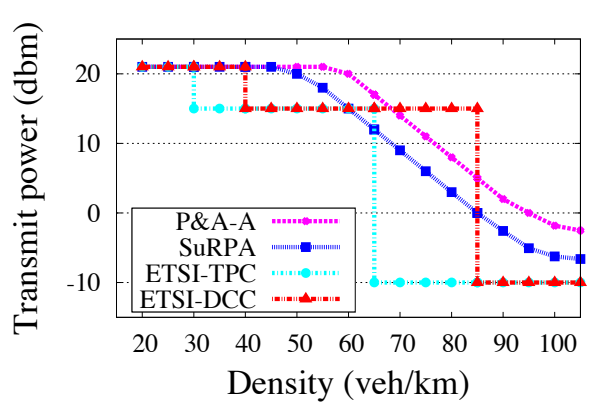

(c) Manhattan

Figure 11: Impact of vehicles density on beacons Transmit Rate and Power parameter: P\&A-A vs. SuRPA and ETSI schemes

the transmission range is higher and the awareness region is larger. Although in some cases Tx parameters are higher in the ETSI schemes than it is in our scheme, this does not come without a cost and it turns out to be very expensive since this compromises the delivery of beacons, as we will show in the next subsection.

\section{P\&A-A: Coupled prediction and adaptation evaluation}

In this subsection, we intend to show the advantage from using our scheme P\&A-A (coupled local density prediction and rate and power adaptation) against the four above-mentioned schemes namely P-SuRPA (including the plain prediction) and the three variants of the ETSI scheme.

Figure 12 reveals the impact of vehicle density on the collision rate and the channel busy ratio in our scheme as well as in all other four schemes. In Figures 12(a) and 12(b), our solution shows the lowest collision rate among all five schemes and is the most stable as this collision rate remains below $8 \%$ in both scenarios during the whole simulation regardless of the density. In contrast, P-SuRPA fluctuates around the threshold of $10 \%$ reaching up to $15 \%$ in higher densities and peaking to $17 \%$ in Manhattan at a density of $100 \mathrm{veh} / \mathrm{km}$. the ETSI schemes reach limits that are not tolerated for VANETs and safety applications in particular with more than $20 \%$ for ETSITRC and ETSI-DCC, and up to $60 \%$ for ETSI-TPC. We also notice a lot of fluctuation for the DCC schemes; this is due to the sharp change in Tx parameters when switching between the DCC states. We refer the reader to [15] for a thorough analysis of the DCC schemes. Similarly, the graphs in Figures 12(c) and 12(d) show the variation of the busy ratio with the increase of density. As these plotted results show, our scheme allows a non-negligible improvement in terms of channel usage as it fluctuates around $35 \%$ in mid and high densities surpassing P-SuRPA and the three other ETSI schemes. We notice that ETSI-TPC reaches approximately the same busy ratio at a density of $100 \mathrm{veh} / \mathrm{km}$ in Manhattan scenario, but this can be discarded since this same scheme has a collision rate higher than 55\% at this density level.

It is crucial here to point out the importance of a low collision rate to achieve a higher level of awareness in vehicular environment. In fact, DCC having higher Tx parameters (Figure 11) for a short period does not mean it achieves a higher awareness level since more beacons are lost (more than twice the value of collision rate than our scheme). That being said, the under utilisation of the channel is not desired either as this channel needs to remain at a good level of utilisation in order to meet the requirements in terms of awareness level. According to our findings presented in Section III, the theoretical maximum channel busy ratio in vehicular networks, that satisfies a marginal collision rate, is around $40 \%$, a value really close to what our protocol achieved.

The next set of results depicted in Figure 13 show the reaction time of each of the considered schemes to the detected changes in channel conditions. Figures 13(a) and 13(b) show the variation of the collision rate after two clusters of vehicles meet (in an intersection for example) for Manhattan and Kirchberg scenarios respectively. We observe a considerable increase in the collision rate of all schemes, then slowly returning to normal rates after a few seconds. While this increase remains very small for our new scheme, it fluctuates to high values: up to $20 \%$ for P-SuRPA and more than $40 \%$ in some of the ETSI schemes. Also, our scheme gets back to its normal rate after approximately 1 second while the four other schemes take a few seconds longer to achieve that. Figures 


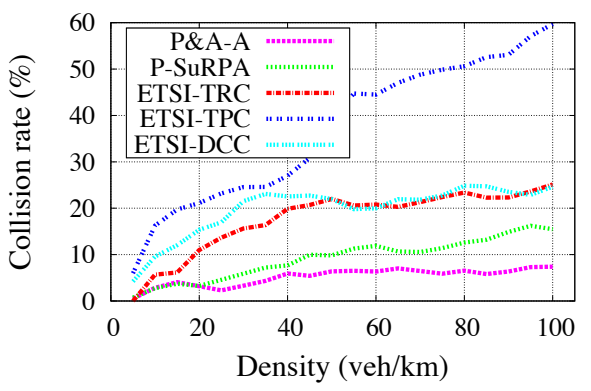

(a) Manhattan

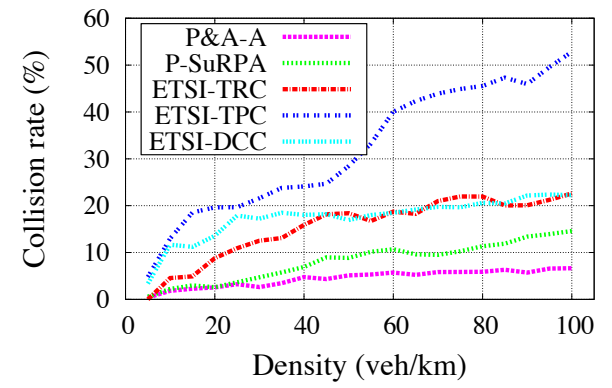

(b) Kirchberg

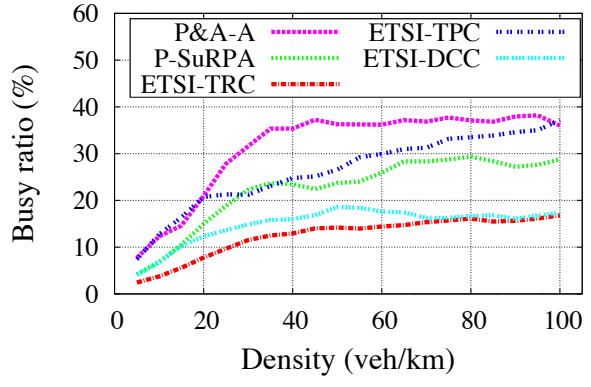

(c) Manhattan

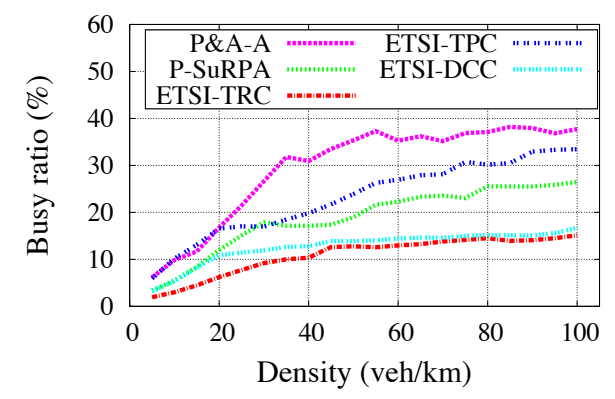

(d) Kirchberg

Figure 12: Impact of vehicles density on the achieved collision rate and channel busy ratio: P\&A-A vs. P-SuRPA and ETSI schemes

13(c) and 13(d) on the other hand show the variation of the busy ratio with time and demonstrates the stability of our new scheme compared to the other schemes as it shows the least variation among all five, while remaining close to the $35 \%$ threshold. The instability of the ETSI schemes is apparent as their respective values rise during second 1 after the vehicles' encounter and drop significantly in the third second due to the sharp adaptation of Tx parameters (see Figure 11).

The previous set of results (Figures 12 and 13) highlight the benefit we obtained after applying our scheme P\&A-A, in terms of network performance. These results are a further validation of our theoretical study conducted in subsection III-B, and prove the effectiveness of the study. It is needless to say how critical it is to achieve good overall network performance, due to the critical nature of applications developed to ensure the safety of drivers on the road. However, we would like to stress on the importance of such good performances in specific times and in a microscopic perspective. In fact, an aggressive reaction to the changes in the network is crucial in VANETs, but this will never be complete without a proper density prediction mechanism to ensure more accurate data about the local density and network state. This is what we have showcased in this section first, by demonstrating the preciseness of our short-term vehicular density prediction; then by displaying the repercussion of this on the performance of the network.

\section{CONCLUSION}

In this paper, we presented P\&A-A, a new solution for channel congestion where beacons' transmit rate and power are adapted successively in order to control the channel load and ensure a better level of awareness among vehicles. This adaptation is performed based on a short-term vehicular local density prediction scheme that aims at providing more accurate and up-to-date information about the network state. This prediction then helps to perform an improved adaptation to the transmission parameters in order to achieve better overall network performance. This modular design allows for easy future improvements of the solution by means of simple software updates. Additionally, we achieved a higher level of awareness by combining rate and power adaptations in a smart way that allows respecting the strict beaconing frequency requirement. Simulations were conducted using a realistic simulation environment in terms of both channel conditions and vehicle mobility, and are fully compliant with the specifications of the IEEE $802.11 \mathrm{p}$ hardware and software. The obtained results have proven the efficiency and the effectiveness of our scheme that enabled a significant enhancement in terms of channel busy ratio and successful packet delivery. The findings of this work will help the research community moving one step forward and gaining deeper understanding of the rate and power adaptation mechanics; and paves the way for more innovative ways to control the channel load in VANETs. It also helps the vehicular networks research community to gain deeper understanding as to why a local density prediction is needed in such a network, and will open new research prospects by bringing up new challenges to VANETs research field.

\section{ACKNOWLEDGMENT}

This work was supported, in part, by Science Foundation Ireland Grant 10/CE/I1855 and by Science Foundation Ireland grant 13/RC/2094 to Lero, the Irish Software Engineering Research Centre (www.lero.ie). This work was also supported 


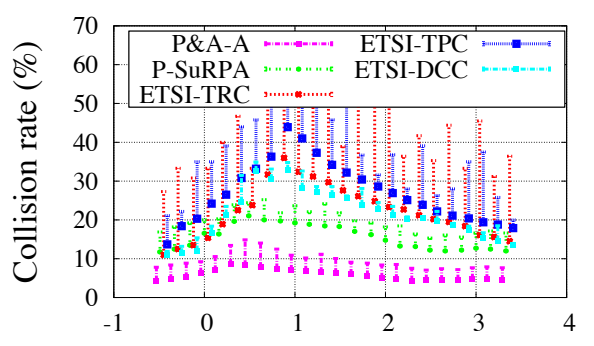

Time after two clusters encounter (s)

(a) Manhattan

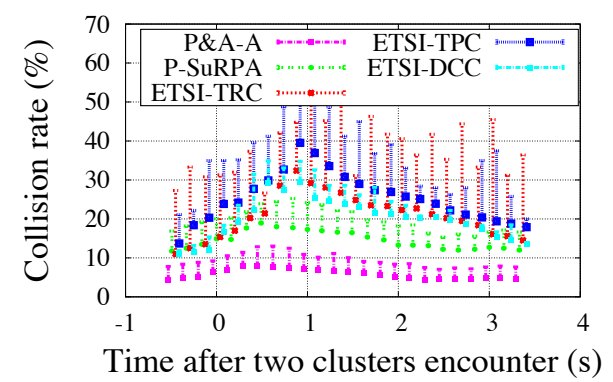

(b) Kirchberg

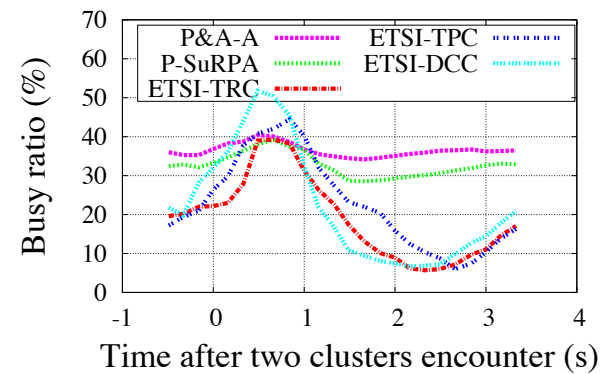

(d) Kirchberg

Time after two clusters encounter (s)

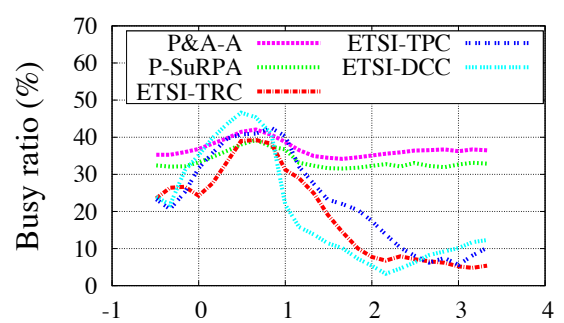

Time after two clusters encounter (s)

(c) Manhattan

Figure 13: Collision rate and channel busy ratio variation after two clusters of vehicles meet: P\&A-A vs. P-SuRPA and ETSI schemes

by the Earth and Natural Sciences Doctoral Studies Programme, which is funded by the Higher Education Authority under the Programme for Research in Third- Level Institutions (PRTLI) Cycle 5 and co-funded under the European Regional Development Fund (ERDF).

\section{REFERENCES}

[1] CAMP Vehicle Safety Communications Consortium. (2005). Vehicle safety communications project task 3 final report: Identify intelligent vehicle safety applications enabled by DSRC, Nat. Highway Traffic Safety Admin., U.S. Dept. Transp. (USDOT), Washington, DC, Tech. Rep. DOT HS 809859. [Online]. Available: http://www.its.dot.gov/research_docs/ pdf/59vehicle- safety.pdf

[2] Park, Y., \& Kim, H. (2013). "Collision control of periodic safety messages with strict messaging frequency requirements", Vehicular Technology, IEEE Transactions on, 62(2), 843-852.

[3] Wireless LAN Medium Access Control (MAC) and Physical Layer (PHY) Specifications Amendment 6: Wireless Access in Vehicular Environments, IEEE, Standard 802.11p-2010, 2010.

[4] ETSI EN 302663 V 1.2.1 Intelligent Transport Systems (ITS). Intelligent transport systems (its); access layer specification for intelligent transport systems operating in the $5 \mathrm{ghz}$ frequency band, 2013.

[5] 1609 WG Dedicated Short Range Communication Working Group 1609.4- 2016 - IEEE Standard for Wireless Access in Vehicular Environments (WAVE) - Multi-Channel Operation, 2016.

[6] Zemouri, S., Djahel, S., \& Murphy, J. (2014, October). "Smart adaptation of beacons transmission rate and power for enhanced vehicular awareness in VANETs", In Intelligent Transportation Systems (ITSC), 2014 IEEE 17th International Conference on (pp. 739-746). IEEE.

[7] C. Sommer, O. K. Tonguz, \& F. Dressler, "Traffic Information Systems: Efficient Message Dissemination via Adaptive Beaconing," IEEE Communications Magazine, vol. 49, no. 5, pp. 173-179, 2011.

[8] C. Sommer, S. Joerer, M. Segata , O. Tonguz, R. L. Cigno, \& F. Dressler, "How shadowing hurts vehicular communications and how dynamic beaconing can help", In Mobile Computing, IEEE Transactions on, 14(7), (2015) 1411-1421.

[9] M. Torrent-Moreno, J. Mittag, P. Santi, \& H. Hartenstein, "Vehicle-tovehicle communication: fair transmit power control for safety-critical information", Vehicular Technology, IEEE Transactions on, 58(7), (2009) 3684-3703
[10] Kloiber, B., Härri, J., \& Strang, T. "Dice the TX power-Improving Awareness Quality in VANETs by random transmit power selection," In VNC, 2012 (pp. 56-63).

[11] Sander Frigau, M. "Cross-layer transmit power and beacon rate adaptation for VANETs," In Proceedings of the third ACM international symposium on Design and analysis of intelligent vehicular networks and applications, Nov. 2013, (pp. 129-136). ACM.

[12] Baldessari, R., Scanferla, D., Le, L., Zhang, W., \& Festag, A. "Joining forces for vanets: A combined transmit power and rate control algorithm," In 6th International Workshop on Intelligent Transportation (WIT), March 2010.

[13] Huang, C. L., Fallah, Y. P., Sengupta, R., \& Krishnan, H. "Adaptive intervehicle communication control for cooperative safety systems," Network, IEEE, 2010, 24(1), 6-13.

[14] ETSI TS 102687 V1.1.1, Intelligent Transport Systems (ITS); Decentralized Congestion Control Mechanisms for Intelligent Transport Systems operating in the $5 \mathrm{GHz}$ range; Access layer part, July 2011

[15] Autolitano, A., Campolo, C., Molinaro, A., Scopigno, R. M., \& Vesco, A. "An insight into Decentralized Congestion Control techniques for VANETs from ETSI TS 102687 V1. 1.1, ” In Wireless Days (WD), Nov. 2013 IFIP(pp. 1-6). IEEE.

[16] Eckhoff, D., Sofra, N., \& German, R. "A performance study of cooperative awareness in ETSI ITS G5 and IEEE WAVE," In Wireless On-demand Network Systems and Services (WONS), 2013 10th Annual Conference on, March 2013, (pp. 196-200). IEEE.

[17] Wu, C., Ohzahata, S., Ji, Y., \& Kato, T. (2016). "How to Utilize Interflow Network Coding in VANETs: A Backbone-Based Approach." IEEE Transactions on Intelligent Transportation Systems, 17(8), 22232237.

[18] Wu, C., Chen, X., Ji, Y., Ohzahata, S., \& Kato, T. (2015). "Efficient broadcasting in VANETs using dynamic backbone and network coding." IEEE Transactions on Wireless Communications, 14(11), 6057-6071.

[19] Zemouri, S., Djahel, S., \& Murphy, J. (2015). "A fast, reliable and lightweight distributed dissemination protocol for safety messages in Urban Vehicular Networks", Ad Hoc Networks, 27, 26-43.

[20] Zemouri, S., Djahel, S., \& Murphy, J. (2013, December). "Short-paper: Road-Casting: A New Distributed Dissemination Protocol for Safety Messages in Urban Areas," in IEEE Vehicular Networking Conference (VNC), Dec. 2013

[21] Sepulcre, M., Gozalvez, J., Altintas, O., \& Kremo, H. (2016). "Integration of congestion and awareness control in vehicular networks", Ad Hoc Networks, 37, 29-43. 
[22] Kenney, J. B., Bansal, G., \& Rohrs, C. E. (2011, September). "LIMERIC: a linear message rate control algorithm for vehicular DSRC systems", In Proceedings of the Eighth ACM international workshop on Vehicular inter-networking (pp. 21-30). ACM.

[23] Tielert, T., Jiang, D., Chen, Q., Delgrossi, L., \& Hartenstein, H. (2011, November). "Design methodology and evaluation of rate adaptation based congestion control for Vehicle Safety Communications", In Vehicular Networking Conference (VNC), 2011 IEEE (pp. 116-123). IEEE.

[24] Soleymani, S. A., Abdullah, A. H., Anisi, M. H., Altameem, A., Hasan, W. H., Goudarzi, S., ... \& Noor, N. M. (2017). "BRAIN-F: Beacon Rate Adaption Based on Fuzzy Logic in Vehicular Ad Hoc Network." International Journal of Fuzzy Systems, 19(2), 301-315.

[25] Aygun, B., Boban, M., \& Wyglinski, A. M. (2016). "ECPR: Environment-and context-aware combined power and rate distributed congestion control for vehicular communications." Computer Communications, 93, 3-16.

[26] Chen, X., Kong, L., Liu, X., Rao, L., Bai, F., \& Xiang, Q. (2016, April). "How cars talk louder, clearer and fairer: Optimizing the communication performance of connected vehicles via online synchronous control." In Computer Communications, IEEE INFOCOM 2016-The 35th Annual IEEE International Conference on (pp. 1-9). IEEE.

[27] Tielert, T., Jiang, D., Hartenstein, H., \& Delgrossi, L. "Joint power/rate congestion control optimizing packet reception in vehicle safety communications," In Proceeding of the tenth ACM international workshop on Vehicular inter-networking, systems, and applications, June 2013, (pp. 51-60). ACM

[28] Namboodiri, V., \& Gao, L. (2007). Prediction-based routing for vehicular ad hoc networks. Vehicular Technology, IEEE Transactions on, 56(4), 2332-2345

[29] Tyagi, V., Kalyanaraman, S., \& Krishnapuram, R. (2012). Vehicular traffic density state estimation based on cumulative road acoustics. Intelligent Transportation Systems, IEEE Transactions on, 13(3), 11561166.

[30] Zemouri, S., Djahel, S., \& Murphy, J. (2015, December). "A short-term vehicular density prediction scheme for enhanced beaconing control", In Global Communications Conference (GLOBECOM), 2015 IEEE

[31] Qian, J., Jing, T., Huo, Y., Li, H., Ma, L., \& Lu, Y. (2016, August). “An Adaptive Beaconing Scheme Based on Traffic Environment Parameters Prediction in VANETs." In International Conference on Wireless Algorithms, Systems, and Applications (pp. 524-535). Springer International Publishing.

[32] 802.11p-2010 - IEEE Standard for Information technology- Local and metropolitan area networks- Specific requirements- Part 11: Wireless LAN Medium Access Control (MAC) and Physical Layer (PHY) Specifications Amendment 6: Wireless Access in Vehicular Environments.

[33] "Standard Specification for Telecommunications and Information Exchange Between Roadside and Vehicle Systems 5 GHz Band Dedicated Short Range Communications (DSRC) Medium Access Control (MAC) and Physical Layer (PHY) Specifications", ASTM E2213-03, 2010.

[34] Y. Park \& H. Kim, "Application-Level Frequency Control of Periodic Safety Messages in the IEEE WAVE" Vehicular Technology, IEEE Transactions on 61.4 (2012): 1854-1862.

[35] Shen W., Djahel S., \& McManis J., "An adaptive and VANETs-based Next Road Re-routing system for unexpected urban traffic congestion avoidance," in IEEE Vehicular Networking Conference (VNC), Dec. 2015.

[36] Balon, N., \& Guo, J. "Increasing broadcast reliability in vehicular ad hoc networks," In Proceedings of the 3rd international workshop on Vehicular ad hoc networks, Sep. 2006, ACM.

[37] A. Mukunthan, C. Cooper, F. Safaei, D. Franklin \& M. Abolhasan, "Studying the Impact of the CORNER Propagation Model on VANET Routing in Urban Environments," in IEEE Vehicular Technology Conference (VTC Fall), Sep. 2012

[38] S. Zemouri, S. Mehar \& S.M. Senouci, "HINTS: A novel approach for realistic simulations of vehicular communications," in Global Information Infrastructure and Networking Symposium (GIIS), Dec. 2012

[39] Jiang, D., Chen, Q., \& Delgrossi, L. (2008, September). "Optimal data rate selection for vehicle safety communications." In Proceedings of the fifth ACM international workshop on VehiculAr Inter-NETworking (pp. 30-38). ACM.

[40] Sommer, C., Eckhoff, D., German, R., \& Dressler, F. (2011, January). "A computationally inexpensive empirical model of IEEE $802.11 \mathrm{p}$ radio shadowing in urban environments." In Wireless On-Demand Network Systems and Services (WONS), 2011 Eighth International Conference on (pp. 84-90). IEEE.

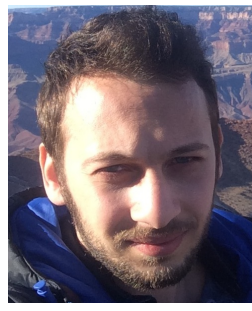

Sofiane Zemouri received the B.S. degree in computer science from the University of Science and Technology Houari Boumediene, Algiers, Algeria; the M.S. degree in telecommunication and computer science from University of Versailles Saint-Quentinen-Yvelines, Versailles, France and Telecom ParisTech, Paris, France, and the Ph.D degree in computer networks performance and engineering from University College Dublin in Ireland. He is currently a Postdoctoral researcher at the Performance Engineering Laboratory group of the Department of Computer Science at University College Dublin, Ireland. His research interests include performance and reliability of communication systems in Vehicular Ad Hoc and Sensor Networks, Software Defined Networks, and Internet of Things.

Soufiene Djahel is a Senior Lecturer at Manchester Metropolitan University (UK) since Sep. 2015 and was an Engineering Research Manager at University College Dublin (Ireland) from Feb. 2012 to Sep. 2015. Before joining UCD, he was a postdoc fellow at ENSIIE (France). He got his Ph.D. degree in computer science in Dec. 2010 from Lille 1 UniversityScience and Technology (France). During his Ph.D., he dealt with several security issues at MAC and Routing layers in wireless multi-hop networks. Prior to that, he spent 6 months at INRIA Nord Europe research center as an engineer researcher working on CLADIS project. He received a Magister degree, with majors in networking and distributed systems, and a State Engineering degree in computer science from Abderrahmanemira University (Bejaia, Algeria) and Badji-Mokhtar University (Annaba, Algeria) in Feb. 2007 and Jun. 2004, respectively. His research interests include Intelligent Transportation Systems (ITS), Security and QoS issues in wireless networks and E-health. He is senior member of the IEEE and reviewer of its major conferences and journals in wireless networks and security. $\mathrm{He}$ was/is the general co-chair of VTM 2014, RA-WERHA 2015 and ISNCC 2016 and the TPC co-chair of VTM 2012, ISNCC 2015 and IEEE ISC2 2016, and has served on the TPC of several IEEE major conferences such as IEEE ICC, IEEE WCNC and IEEE Globecom.

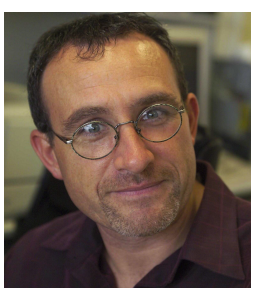

John Murphy is an Associate Professor in Computer Science and Informatics at University College Dublin. He got a first class honours degree in electronic engineering (B.E.) in 1988 from the National University of Ireland (UCD), an M.Sc. in electrical engineering from the California Institute of Technology in 1990 and a Ph.D. in electronic engineering from Dublin City University in March 1996. He is an IBM Faculty Fellow, a Senior Member of the IEEE, a Fellow and Chartered Engineer with Engineers Ireland, and a Fellow of the Irish Computer Society. For many years he held an academic part-time position at the Jet Propulsion Laboratory in Pasadena, and acted as a consultant to the US Department of Justice. Prof. Murphy is an associate editor for both IEEE Communications Letters Journal, a member of the Editorial Board for the IET Communications (formerly IEE Proc Communications), and an associate editor for Telecommunications Systems Journal. He was the guest editor (along with Prof. Perros) for a special issue of IET Communications on Optical Burst and Packet Switching in 2009. He has published over 100 peer-reviewed journal articles or international conference full papers in performance engineering of networks and distributed systems and has been awarded over 20 competitive research grants (over 5 million euro). He has supervised $13 \mathrm{Ph} . \mathrm{D}$. students to completion. 